\title{
Review article: Contribution of magnetic resonance imaging to the management of esophageal diseases: a systematic review
}

Authors: Chloé Leandri ${ }^{1,2}$, MD, Philippe Soyer ${ }^{2,3}, \mathrm{MD}, \mathrm{PhD}$, Ammar Oudjit ${ }^{3,}$ MD, MarieAnne Guillaumot ${ }^{1}, \mathrm{MD}$, Stanislas Chaussade ${ }^{1,2}, \mathrm{MD}, \mathrm{PhD}$, Anthony Dohan ${ }^{2,3}, \mathrm{MD}, \mathrm{PhD}$, Maximilien Barret ${ }^{1,2}, \mathrm{MD}, \mathrm{PhD}$.

${ }^{1}$ Gastroenterology Department, Cochin University Hospital, Assistance Publique-Hôpitaux de Paris, France

${ }^{2}$ Paris Descartes University, Paris France,

${ }^{3}$ Department of Radiology, Cochin University Hospital, Assistance Publique-Hôpitaux de Paris, France

Corresponding author:

Maximilien Barret, $\mathrm{MD}, \mathrm{PhD}$.

Cochin Hospital, Gastroenterology Department

27, rue du Faubourg St Jacques, 75014 Paris, France.

E-mail: maximilien.barret@aphp.fr

Tel: +33(1) 58414285

Fax: +33(1)58412836

Chloé Leandri (chloe.leandri@aphp.fr): acquisition of data; analysis and interpretation of data; drafting of the manuscript

Philippe Soyer (philippe.soyer@aphp.fr): critical revision of the manuscript for important intellectual content

Ammar Oudjit (ammar.oudjit@aphp.fr): critical revision of the manuscript for important intellectual content

Marie-Anne Guillaumot (marie-anne.g@hotmail.fr): acquisition of data; analysis and interpretation of data

Stanislas Chaussade (stanislas.chaussade@aphp.fr): critical revision of the manuscript for important intellectual content

Anthony Dohan (anthony.dohan@aphp.fr): acquisition of data; critical revision of the manuscript for important intellectual content

Maximilien Barret (maximilien.barret@aphp.fr): study concept and design, study supervision

Declarations of interest:

non 


\section{Abstract:}

Purpose: Currently available imaging modalities used to investigate the esophagus are irradiating or limited to the analysis of the esophageal lumen. Magnetic resonance imaging (MRI) is a non-invasive and non-radiating imaging technique that provides high degrees of soft tissue contrast. Newly developed fast MRI sequences allow for both morphological and functional assessment of the esophageal body and esophagogastric junction. The purpose of this systematic review was to identify the contribution of MRI to the diagnosis and management of esophageal diseases, such as gastroesophageal reflux, esophageal motility disorders, esophageal neoplasms, and portal hypertension. Methods: We performed a systematic search of the Medline (via Ovid), EMBASE (via Ovid), PubMed and Cochrane Library databases from inception to December 2018 inclusively, using the MESH major terms "magnetic resonance imaging" AND "esophagus". Results: The initial search retrieved 310 references, of which 56 were found to be relevant for the study. References were analysed and classified in different subheadings: MRI protocols for the esophagus, gastroesophageal reflux disease, achalasia and other esophageal motility disorders, esophageal cancer, portal hypertension and other esophageal conditions. Conclusion: MR Esophagography might become a non-invasive, non-irradiating technique of choice following diagnostic esophagogastroduodenoscopy for the assessment of esophageal diseases.

Keywords: Esophageal motility disorders; Gastroesophageal reflux disease; Esophageal neoplasm; Magnetic resonance imaging (MRI) 


\section{INTRODUCTION}

Esophageal diseases are frequent conditions in adult and include a variety of benign or malignant diseases, all with serious potential impact on the quality of life. Symptoms suggestive of esophageal disease include dysphagia, regurgitations, heartburn, and retrosternal pain. Malignant conditions include esophageal neoplasia, epithelial or subepithelial tumors. Benign diseases include esophageal motility disorders, gastro-esophageal reflux disease, esophageal involvement of systemic or dermatologic diseases (scleroderma or lichen planus), and morphological abnormalities such as esophageal diverticula and esophagitis from various causes.

Upper gastrointestinal endoscopy is currently the first step for the diagnostic workup of patients with symptoms suggestive for esophageal disease because of its ability to rule out an esophageal stenosis, and identify a large number of esophageal diseases, either optically or by histopathological analysis of tissue samples obtained by mucosal biopsy (1). However, endoscopy is an invasive technique, often requiring sedation, thereby increasing its costs with potential morbidity (2). Furthermore, additional diagnostic examinations are further needed when upper gastrointestinal endoscopy does not help making a definite diagnosis. Endoscopic ultrasonography allows for a detailed study of the esophageal wall and periesophageal region, searching for esophageal intramural or regional lesions, but is limited by its availability, the need for sedation, its costs, and its poor diagnostic contribution in patients with esophageal stenosis or widely dilated esophagus. Esophageal functional testing by manometry is the standard of care for patients with esophageal motility disorders. However, esophageal manometry provides information regarding pressure pattern but little morphological information. Thus, an esophageal or periesophageal lesion may be misdiagnosed as an esophageal motility disorder. $\mathrm{PH}$ or $\mathrm{pH}$-impedance measurement can document gastroesophageal reflux disease, but at the penalty of a $24 \mathrm{~h}$ long investigation, and without any morphological information. Finally, the development of tailored endoscopic treatments for esophageal motility disorders using endoscopic esophageal myotomy urges the need for an optimal morphological assessment of the esophagus and esophagogastric junction.

Oral barium esophagogram may reveal morphological endoluminal abnormalities, such as diverticula, esophageal stenosis, hiatal hernia or megaesophagus, as well as motility disorders through dynamic acquisition such as video-fluoroscopy. However, it does not provide much information on the periesophageal tissue, and its use is restricted, owing to the 
exposure to ionizing radiation. Computed tomography (CT) is mainly indicated in the setting of esophageal neoplasia, in order to rule out distant metastases, or major loco regional tumoral spread. It may be useful, with oral opacification and intravenous administration of iodinated contrast material, to search for postoperative complications, particularly anastomotic leaks $(3,4)$. However, CT is a radiating diagnostic modality and repeated acquisition causes increased radiation exposure.

MRI is not a routine examination for the assessment of esophageal diseases, mainly because of its relatively low availability and its technical limitation. Indeed, the vicinity of air containing organs such as the esophagus itself, but also the stomach, lungs and trachea may alter seriously the quality of the images. Furthermore, motion due to respiration and heart beating add to the challenges of making sharp depiction of the esophagus. However, MRI is non-invasive, non-radiating, and provides an excellent soft-tissue contrast (5). Dynamic MRI sequences provide information regarding the motility of the oesophagus.

The purpose of this systematic review was to identify the contribution of MRI to the diagnosis and management of esophageal diseases, such as gastroesophageal reflux, esophageal motility disorders, esophageal neoplasms, and portal hypertension.

\section{MATERIALS AND METHODS}

\section{Search strategy}

This systematic review was conducted using a pre-specified protocol and was guided by the Preferred Reporting Items for Systematic Reviews and Meta-Analyses (PRISMA) checklist (6). We performed a systematic search of the Medline (via Ovid), EMBASE (via Ovid), PubMed and Cochrane Library databases from inception to December 2018 inclusively, using the MESH major terms "magnetic resonance imaging" AND “esophagus". Moreover, published reviews, case reports and case series were manually searched for additional studies. After exclusion of duplicates, the titles and abstracts of publications identified by the database search were screened for studies that potentially met the inclusion criteria. This screening was independently performed by two of the authors (C.L. and M.B.). A third reviewer resolved all disagreements (A.D.). 


\section{Study eligibility}

Full-text publications were reviewed to determine final eligibility. Eligibility was restricted to original studies detailing esophageal MRI protocol and esophageal diseases. Articles pertaining to pediatric, cardiovascular, and otorhinolaryngologic diseases were excluded. Only articles published in English language literature were included in our study. Commentaries, abstracts, reviews articles and conference presentations were excluded.

\section{RESULTS AND DISCUSSION}

The initial search retrieved 310 references, of which 56 were found to be relevant for the study. We excluded four literature reviews on MRI and esophageal diseases [5, 7, 8, 9]. One was focused on the role of MRI-guided radiotherapy for esophageal and gastric neoplams (7). Another review specifically studied the role of MRI to assess tumor response to neoadjuvant chemoradiotherapy (8). In another work focusing on radiological imaging of the lower esophageal sphincter, Sharitzer et al. concluded that MRI might be of help to identify structural abnormalities in the reflux barrier of patients with gastro-esophageal reflux disease and to assess the esophagogastric function with three-dimensional (3D) image analysis (5). The fourth review studied MRI for the imaging of hypopharynx and cervical esophagus and found that MRI was better than CT to assess the esophageal verge and cervical esophagus, because of better soft tissue delineation and less obscuration of this area by beam-hardening artefacts caused by the shoulders (9).

\section{MRI protocols for the esophagus (Figure 1)}

The normal appearance and measurements of the esophagus in the sagittal plane were first assessed in 78 patients using ECG-gated MRI by Nakashima et al. (Figure 2A) (10). Other authors performed MRI studies on human cadavers with pre-treatment motion-triggered MRI in patients to precisely describe the anatomy of the periesophageal connective tissue layers, (11). Another study on human cadavers using high-resolution MRI with an external surface coil placed over the thorax, and axial T2-weighted fast spin-echo (FSE) images from the superior aspect of the aortic arch to the gastric cardia helped define the MRI anatomy of the posterior mediastinum (12). The specific value of sagittal images to visualize bolus contrast 
and peristalsis was also underlined by most of the publications of the field, particularly during dynamic MR imaging of the esophagus (Figure 2B) (13).

Manabe et al. compared T1-weighted ultrafast gradient echo (TFE, Philips, Best, The Netherlands) MR images and T1-weighted fast field echo (FFE) MR images in 20 healthy volunteers (14). These researchers showed that T1-weighted FFE images provided better signal-to-noise ratio, better image quality, and less artefacts than T1-weighted TFE images (14). Furthermore, they found that $35-\mathrm{mm}$ slice thickness was optimal to delineate the esophageal wall during MR fluoroscopy (14). However, this study was performed in 2004 and several technical refinements have been achieved since that time. The use of an external surface coil and cardiac gating also helped improve the signal of the esophageal wall, as demonstrated by Ridell et al. in 4 healthy volunteers using T2-weighted FSE sequences (15). These authors obtained 22 axial images of the esophagus in an acceptable scan time of 7 min 30s (15).To better evaluate the esophageal wall, intraluminal administration of contrast agents has been tested in several studies $(13,15-18)$. In one study, $100 \mathrm{~mL}$ of a ferric ammonium citrate-cellulose paste were orally administered to 38 patients with esophageal diseases and 10 healthy subjects who served as controls resulting in excellent esophageal opacification in $84 \%(32 / 38)$ of examinations (16). Other researchers tested a home-made mixture made of gadopentetate dimeglumine and barium in 53 patients who underwent T1-weighted MRI resulting in esophageal opacification in 97\% (37/38) of patients with esophageal diseases and $66 \%(10 / 15)$ of control subjects (17). These orally administered contrast agents were reported as easy to use, well-tolerated and did not produce artefacts $(13,15-18)$. Kulinna-Cosentini et al. used a gadolinium-chelate mixed with buttermilk to assess the gastroesophageal junction by MRI in 20 healthy volunteers with dynamic gradient-echo (GRE) sequences (balanced fast field echo [B-FFE]) in three planes on a $1.5 \mathrm{~T}$ unit using a phased-array body coil (13). They reported a mean visible length of the esophagus of $16.2 \mathrm{~cm}$ in the sagittal plane, and defined normative values for the bolus transit time, from the pharyngo-esophageal sphincter region to the gastroesophageal junction. This contrast agent seems advisable, since the same researchers used it in further studies on the same topic (19).

Curcic et al. have described a dynamic MRI protocol to assess the structure and function of the esophagogastric junction and identify reflux in real time in 12 healthy volunteers, after ingestion of a solid and a liquid labeled meal (20). This protocol consisted of a balanced FFE sequence providing 30 images in the transverse plane covering the 
esophagogastric junction area during inspiration and expiration. Then, they performed dynamic MR imaging using three parallel coronal oblique image sections with a B-FFE sequence centred on the esophagogastric junction, yielding 380 dynamic images. Finally, they obtained three-dimensional reconstructions of the esophagus and proximal portion of the stomach, allowing measuring the distribution of angles around the esophagogastric junction. Using an echo navigator method, they were able to detect gastro-esophageal reflux episodes $(20,21)$. This MRI protocol was further used in two subsequent studies $(22,23)$. Figure 3 shows MR images of the gastroesophageal junction in a patient with pseudoachalasia caused by a tight fundoplication.

A recent study reported the use of concentrated pineapple juice mixed with potatoe starch an as intraluminal agent for MRI examination of the esophagus. This mixture allowed a feasible and safe esophageal visualisation, with a patient acceptability and an image quality similar to paramagnetic contrast agents (24).

\section{MRI of the esophagus (how we do it?)}

In our referral tertiary center, the MRI protocol includes T2-weighted single shot spine echo sequences in the axial and coronal plane, dynamic kinematic acquisition of steady-states sequences in the oblique plane, parallel to the esophagogastric junction, and T1-weigthed images in the coronal and axial planed before and after intravenous administration of a gadolinium chelate (Figure 4). Patients swallow water through a straw during kinematic acquisition, which is repeated several times in order to visualize the contractions of esophagus and the impact of extra-luminal lesions on the lumen. The sequences parameters are reported in table 1.

Correct placement of sagittal oblique kinematic sequences is critical to obtain a good visualisation of esophagus and esophagogastric junction as the heart may also be in the acquisition box and lead to motion artefacts. Morphological signs are analysed on T2 weighed images and steady state cine sequences.

\section{Gastroesophageal reflux disease (GERD)}

The esophagogastric junction is an integrated sphincter complex, which includes the lower esophageal sphincter and the crural diaphragm. The wideness of the esophagogastric junction, 
the opening of the esophago-gastric insertion angle or angle of His and the presence of a hiatus hernia are anatomical data that can promote gastro-esophageal reflux.

Kulinna-Cosentini et al. have studied the esophagogastric junction by dynamic MRI in 20 healthy volunteers (13). They reported the imaging features of hiatal hernia, the effect of the Valsalva manoeuver, and gastro-esophageal reflux (13). In a prospective study on 37 patients with GERD symptoms undergoing MRI swallowing in the supine position at $1.5 \mathrm{~T}$ with a phased-array body coil, and subsequent manometry and $24 \mathrm{~h} \mathrm{pH}$ monitoring, KulinnaCosentini et al. found a sensitivity of $82 \%$ (23/28) for the diagnosis of GERD. Furthermore, the size of the hiatal hernia and the presence of gastroesophageal reflux assessed by MRI was significantly correlated with the esophageal acid exposure on $24 \mathrm{~h} \mathrm{pH}$-monitoring (18).

Other researchers tested the hypothesis that dynamic MRI could show the structural factors of the esophagogastric junction thought to be impaired in patients with GERD (23). These authors used three-dimensional models from dynamic MRI in 24 healthy volunteers and 24 patients with GERD. Patients were studied in the right decubitus position to reduce transient relaxation of the lower esophageal sphincter after a test meal. MRI-detected reflux events were also confirmed by the relaxation of the lower esophageal sphincter assessed by concurrent esophageal manometry. They found that the His angle was wider in patients with GERD than in control subjects $\left(+7^{\circ} \pm 3^{\circ} ; P=0.03\right)$. Similarly, the esophagogastric junction more open during reflux events in patients with GERD $(19.3 \mathrm{~mm})$ than in control subjects $(16.8 \mathrm{~mm})(\mathrm{p}=0.04)$. Other esophagogastric morphologic changes, such as position of insertion and gastric orientation within the abdomen, or short intra-abdominal length of the lower esophageal sphincter were also different in patients with GERD and in control subjects. In this study however, the number of reflux events diagnosed by MRI was not different between GERD with patients (3; $95 \%$ CI: 2.5 - 4.6) and controls (2; $95 \%$ CI: $1.8-3.3)(\mathrm{p}=$ 0.09) (23).

MRI appears as a promising tool for the assessment of anti-reflux surgical procedures. In a study including 29 patients with Nissen fundoplication, among which 21 were symptomatic, Kulinna-Cosentini et al. identified the position of fundoplication in 27/29 patients $(93 \%)$, and wrap malfunctions, such as malposition in 4/6 patients (33\%), wrap disruption in 4/14 patients (29\%), stenosis and secondary motility disorders in 8/8 patients (100\%) (10). In a further study with 79 patients, the same group addressed the inter-observer 
variability of MRI (25). In the study, four independent readers, among which two surgeons, evaluated HASTE MR Images obtained at 3.0-T for anatomical assessment of the gastroesophageal junction followed by dynamic MR swallowing (fast low-angle shot sequences). They found a substantial agreement for recurrent hiatal hernia $(\mathrm{k}=0.703)$, moderate agreement for wrap disruption $(\mathrm{k}=0.585)$, and fair agreement for motility disorder and slipping ( $\mathrm{k}=0.234$ and $\mathrm{k}=0.200$, respectively). Overall, MR swallowing, even with nonexperienced readers, helps depict the cause of failed antireflux surgery (25).

\section{Achalasia and other esophageal motility disorders}

The workup of esophageal motility disorders typically includes upper gastrointestinal endoscopy with mucosal biopsies, followed if normal by high resolution manometry. Manometry can however be inconclusive, in case of impossibility to cross the esophagogastric junction with the manometry catheter due to massive esophageal distension, esophageal diverticulum, hiatal hernia, or extremely tight lower esophageal sphincter. In these situations, barium esophagogram is performed to evidence a megaesophagus and a bird's peak appearance at the esophagogastric junction, findings suggestive of achalasia. In patients with atypical presentation such as advanced age, short duration of symptoms, important weight loss and difficulty to cross the esophagogastric junction during endoscopy, additional investigation such as endoscopic ultrasonography or CT is advised to rule out a malignancyassociated pseudoachalasia (26). Therefore, MRI might play a role in this clinical setting, if it is able to bring information on the esophageal motility and possible distension, and on the periesophageal area.

In patients with achalasia, MRI features include continuous dilatation of esophagus with stasis of food residues and liquid with a diffuse mild wall thickening and absence of lower esophagus sphincter (LIS) opening. Panebianco et al. showed that functional MRI can reveal typical esophageal motility disorders $(27,28)$. These researchers performed functional MRI with dynamic T1-weighted turbo-fast low-angle shot sequences during bolus of food mixed with gadolinium-diethylene triamine pentaacetic acid (Gd-DTPA). All 14 patients with motility disorders assessed by fluoroscopy and manometry were also correctly identified at functional MRI to have achalasia ( 9 patients, 6 with a megaesophagus and 3 with early stage achalasia) or esophageal spasm (6 patients). In patients with achalasia, they observed a narrowing of the distal esophagus with a retro dilatation of the proximal segment, a poor 
relaxation of the esophagogastric junction, and an absent peristalsis with increased bolus time transit $(27,28)$. In patients with oesophageal spasm, an intermittent progression of the contrast bolus with tertiary peristalsis, an increased transit time and a reduced calibre with a “corkscrew" appearance was reported $(27,28)$.

Covotta et al. described morphological and functional MRI features of the esophagus in patients with motility disorders. Using T2-weighted HASTE and T1-weighted turbo FLASH sequence, they compared the MRI findings of 24 patients with dysphagia and motility disorders diagnosed by manometry and fluoroscopy to those of 8 healthy control subjects. They found that an ineffective or absent peristalsis on MRI is significantly associated with a slower transit time and an increased esophageal diameter (29).

Miyazaki et al. suggested that functional MRI could diagnose achalasia similar to manometry. Eleven patients with suspected achalasia and 3 healthy patients without dysphagia underwent functional MRI during clear-liquid swallowing with T2-weighed single shot fast spin-echo (SSFSE) and fast imaging employing steady-state acquisition (FIESTA). They defined the luminal fluctuation index (LFI) to study the peristalsis by the formula LFI = Rmax (maximal diameter ratio) x SDratio (standard deviation value). To study the relaxation of the lower esophageal sphincter, they defined Dd and Ds as maximal and minimal diameters at this level. Functional MRI revealed achalasia in 10/11 patients (91\%); MRI findings included tortuous $(10 / 11 ; 91 \%)$ or dilated $(9 / 11 ; 82 \%)$ esophagus without obstructive lesion, poor bolus clearance $(10 / 11 ; 91 \%)$, simultaneous contraction waves $(9 / 11 ; 82 \%)$, aperistalsis $(11 / 11 ; 100 \%)$, and impaired relaxation of the lower esophageal sphincter (7/7) (30). These promising results were however not reproduced by other teams. Indeed, a widely dilated esophageal body is the only radiological sign reported by most other MRI studies in achalasia patients (18).

The main differential is pseudo-achalasia for which MRI provides a better visualization of oesophagus wall and allows a direct visualization of the exoluminal lesions, while endoscopy and barium esophagogram only provide an indirect visualization of the obstacle and show a narrowing of the lumen. MRI also provides a direct visualization of modified anatomy after surgery such as fundoplication. 


\section{Esophageal cancer}

\section{A- MRI for esophageal cancer staging}

An optimal and repeated imaging modality of the esophagus is needed for the initial diagnostic workup, radiotherapy centering, follow-up of patients with esophageal cancer and possibly prediction of response to therapy. Currently, upper gastrointestinal endoscopy and endoscopic ultrasonography are limited by the high prevalence of esophageal stenosis whereas CT has limited capabilities for the assessment of intraluminal lesions. Few clinical studies are available in this field. Representative MRI findings of an esophageal adenocarcinoma are presented in Figure 5.

In one study, MRI examination of the esophagus was feasible in 54 patients with thoracic esophageal squamous cell carcinoma, with a sensitivity for the diagnosis of cancer of $81 \%$ (44/54) (31). The normal esophagus and the esophageal tumor had similar signal intensities on T1- and T2-weighted images. Tumors were mostly visible on T1-weighted sagittal images. The antero-posterior diameter of the tumors correlated well with that found at histopathological analysis of resected specimens. Only T3 and T4 tumors larger than $30 \mathrm{~mm}$, were visible (31).

In an ex-vivo study at $3 \mathrm{~T}$ including 32 resected esophagus with carcinoma, Wei et al. compared the results of MRI-based T staging with histopathological findings. MRI allowed a correct $\mathrm{T}$ staging in 29/32 tumors (90.6\%). The accuracy was $93.8 \%, 90.6 \%, 96.9 \%$, and $100 \%$ for diagnosing an infiltration of the mucosa, submucosa, muscularis propria, and adventitia, respectively (32). Using a 4.5-T MRI, Yamada et al. studied in a similar study setting 70 esophageal squamous cell carcinomas. Depth of extension found at MRI was correctly evaluated in 66/70 tumors (94\%) as confirmed by histopathological analysis (33).

Similar results were found by Stoker et al. who reported a correct T staging in 8/9 esophageal specimens (89\%). However, in this study 60\% (56/91) of metastatic lymph nodes were missed at MRI (34). A more recent study demonstrated, on 20 esophageal specimens with esophageal cancer, the feasibility of diffusion-tensor MR imaging at an ultra-high field strength (7.0T), and the possibility to adequately identify eight tissue layers in the normal esophagus: epithelium, lamina propria, muscularis mucosae, submucosa, inner circular muscle, intermuscular connective tissue, outer longitudinal muscle, and adventitia (35). When compared to histopathology, MRI allowed for a $100 \%$ accurate $\mathrm{T}$ staging, including the differentiation between T1a and T1b stages in esophageal cancer specimens (36-38). 
Koyama et al. studied 13 patients with esophageal cancer with cine-MRI while chewing gum, using steady state sequences (trueFISP, Siemens Healthinners). They found that T3 or T4 esophageal cancers stage were associated with a complete or partial loss of peristalsis on MRI (39).

In a pilot study, Riddell et al. included 10 patients with confirmed oesophageal carcinoma who underwent MRI on the day before surgery (40). They confirmed in vivo the first ex vivo data from Yamada et al. and showed that the external surface coil MRI with T2weighted sequences showed detailed elements of esophageal layer. Abnormal signal intensity interpreted as tumor was histologically confirmed in only $26 \%(13 / 50)$ of tumor MRI images, while only fibrotic changes were found in 56\% (28/50) of tumor MRI images, and no tumor was found on the last $18 \%$ (9/50). Despite these disappointing results, the authors concluded that esophageal MRI could be an alternative technique of local staging of oesophageal cancer before surgery (40). Disappointing were also the results from a study focusing on the T2* value of 48 patients with esophageal squamous cell carcinoma. The authors found that this variable allowed discriminating between $\mathrm{T} 1$ stages from other T stages, but not T2 from T3, or any $\mathrm{N}$ stage (41). $\mathrm{T} 2 *$ value reflects the contents in deoxygenated hemoglobin of an organ. Since the proliferation of malignant tumor cells require oxygen, major cell proliferation in both T2 and T3 tumors should lead to a significant decrease in deoxyhemoglobin in these lesions, without noticeable difference between the $\mathrm{T} 2^{*}$ values of stages $\mathrm{T} 2$ and $\mathrm{T} 3$ tumors. Recently, Wu et al correlated gross tumor volume assessed on T2-weighted imaging, contrastenhanced T1-weighted and diffusion-weighted MRI (DWI) with T and N stage in 60 patients with esophageal squamous cell carcinoma (42).They found that the gross tumor volume is associated with the $\mathrm{T}$ stage and the presence of lymph node metastases. Also, they reported that the gross tumor volume diagnosed on contrast-enhanced T1-weighted imaging better predicts the $\mathrm{T}$ stage, while DWI with a $\mathrm{b}$ value of $500 \mathrm{~s} / \mathrm{mm}^{2}$ better predicts the presence of lymphatic metastasis (42). Studies suggested that a pathological apparent diffusion coefficient (ADC) on diffusion-weighted MRI could be a potent prognosis factor. Giganti et al. investigated the potential prognostic role of $\mathrm{ADC}$ in 23 patients with esophageal or oesogastric junction cancer. ADC values $\leq 1.4 \times 10^{-3} \mathrm{~mm}^{2} / \mathrm{s}$ were significantly associated with a negative prognosis both in the total population and in the surgery-only group $(43,44)$. In another study on 73 patients with locally advanced esophageal squamous cell carcinoma only treated with chemoradiotherapy, Liu et al. found that $\mathrm{ADC} \leq 1.076 \times 10^{-3} \mathrm{~mm}^{2} / \mathrm{s}$ predicted significantly worse survival (45). 
To our knowledge, only one study has prospectively compared diffusion-weighted MRI to endoscopic ultrasound, CT, and 18-FDG PET/CT for preoperative esophageal cancer staging. In the study, the authors found similar accuracy for MRI, EUS and CT for T staging (83\%, 78\% and 78\%; $\mathrm{p}=\mathrm{NS}$ for all comparisons), and $\mathrm{N}$ staging $(66 \%, 50 \%$ and $61 \%$; $\mathrm{p}=$ NS for all comparisons) (46). Noticeably, in this study, the impact of PET/CT could not be assessed because only one out of the 4 patients with lymph node metastases exhibited a nodal uptake. However, this work paves the way for a potential change in the pretherapeutic workup of esophageal cancer, in which MRI with DWI could replace more costly or invasive tests such as PET/CT or endoscopic ultrasound.

\section{B- MRI for follow-up during and after treatment}

MR-guided stereotactic radiation therapy enables daily imaging without exposing patients to additional radiation. The application of this technique to the esophagus however requires taking into account the peristaltic movements of the esophagus, as proposed in a preliminary study by Cardenas et al. (47). Similarly, other studies involving 33 consecutive patients with esophageal cancer investigated with cine MRI described the motion of esophageal tumors in the anterior-posterior, superior-inferior, and left-right directions, in order to optimise the gating of the radiotherapy $(48,49)$.

The assessment of the metabolic activity of a tumor by diffusion-weighted MRI could be a promising contribution of MRI to the management of esophageal cancer, particularly in terms of follow-up after neoadjuvant or definitive chemoradiotherapy (50-58). Liu et al. studied the diffusion weighted MR images of 46 patients with esophageal SCC of which 18 $(18 / 46 ; 39 \%)$ were treated by chemoradiotherapy and 38 (38/46) were operated on. They found the $\mathrm{b}$ values of $800 \mathrm{~s} / \mathrm{mm} 2$ and $1000 \mathrm{~s} / \mathrm{mm} 2$ to be optimal for observing the efficacy of chemoradiotherapy, while a b value of $600 \mathrm{~s} / \mathrm{mm} 2$ is advisable to measure the length of esophageal squamous cell carcinoma (59). In a recent meta-analysis of 7 studies including a total of 236 patients treated by chemoradiotherapy, the pooled sensitivity and specificity of MRI for predicting early response to chemo-radiotherapy were 93\% (95\% CI: 77\%-98\%) and $85 \%$ (95\% CI: $72 \%-93 \%$ ) for the $\Delta$ apparent diffusion coefficient (ADC) respectively; and 
75\% (95\% CI: 62\%-84\%) and 90\% (95\% CI: 67\%-97\%) for the post-ADC, respectively (60). These data from small studies with heterogeneous clinical settings (neoadjuvant and definitive chemoradiotherapy) suggest however that diffusion-weighted MRI has acceptable performances for assessing the response of patients with esophageal cancer to chemoradiotherapy. Heethuis et al also suggested, in a study including 12 patients, that dynamic contrast enhanced MRI was also of help in predicting histopathologic response to neoadjuvant chemoradiotherapy for oesophageal cancer. Indeed, the change in tumor areaunder-the-concentration time curve had a sensitivity of $83 \%$, specificity of $88 \%$, positive predictive value of $71 \%$, and a negative predictive value of $93 \%$ for predicting histopathologic complete response to neoadjuvant chemoradiotherapy (61). Currently, a prospective multicentre trial is on-going to assess complete pathological tumor response of esophageal cancer after neoadjuvant chemoradiotherapy with MRI, and possibly avoid unnecessary esophagectomy (62). In Table 2, we reported b-values used for diffusion weightimaging in different studies.

While MRI-guided radiotherapy remains under development (7), MR esophagography with water swallowing was promising in assessing the response to radiotherapy (63). In a study including 30 patients with thoracic esophageal carcinoma treated with surgery or chemoradiotherapy and 10 healthy volunteers, Zhang et al. compared the tumor imaging by conventional MRI (T1-weighted spin echo, T2 weighted axial turbo spin-echo or T2-weighted HASTE sequences) and water swallowing MR esophagography (steady state sequences) (60). They observed the resolution of the neoplastic esophageal stenosis and the periesophageal mass in all patients under chemoradiotherapy (60). Furthermore, MRI esophagography with water swallowing was better than conventional MRI for assessing tumor length (88\% vs $12 \%$, respectively) and exact localization (100 vs. 80\%; respectively), but less accurate in terms of T tumor staging (60).

After esophagectomy and narrow gastric-tube reconstruction, Panebianco et al. showed that MRI could be a useful radiation-free imaging modality to investigate the functionality of neo-esophagus. MRI with steady state and turbo-FLASH T1-weighted sequences during oral administration of yoghurt mixed with gadolinium based oral contrast agent was obtained in 10 patients 1 to 4 years after surgery. The authors identified completely re-established motility of the neo-esophagus in $3 / 10$ patients, mild to moderate alterations 
including raised transit time, reflux and contrast agent stasis in 6/10 patients, and 1/10 patient with severe alterations of the contractility a grossly dilated neo-esophagus, severe reflux and stasis (64).

\section{Portal hypertension}

One study evaluated oesophageal mural veins and paraesophageal veins by MR portography in patients with portal hypertension. The authors included 57 patients, with endoscopically documented esophageal varices and found a significant correlation between the diameter of the esophageal mural veins assessed by MR angiography and the endoscopic grade of the esophageal varices $(r=0.363 ; p=0.02)(65)$. However, no other team has reproduced these results ever since.

\section{Other esophageal conditions}

Several systemic and dermatologic conditions may affect the esophagus. In a prospective study including 13 patients with systemic sclerosis, Sauter et al. assessed the impact of wholebody PET/MRI (66). While positron emission tomography (PET) scanning allowed differentiating limited from diffuse disease through the fascial fluorodesoxyglucose (18 FDG) uptake, MRI accurately identified esophageal involvement of the disease (66).

\section{CONCLUSION}

During the last two decades, technical refinements in MRI have allowed to assess not only the morphology of the esophagus and esophagogastric junction, but also the motility and function of the esophagus during swallowing and gastro-esophageal reflux. Overall, publications in the field are scarce and limited to preclinical studies or small pilot studies. While it has become feasible to image the esophagogastric junction with MRI, the diagnostic of gastroesophageal reflux disease based on MRI study does not seem to be reproducible and reliable yet. The 
contribution of MRI to the field of esophageal motility disorders is still limited, but likely to increase, given the suboptimal assessment of the esophageal morphology currently available with endoscopy, barium esophagogram and CT scanner. Further studies, reporting MR features of the main esophageal motility disorders defined by the latest Chicago classification v3.0, are needed. Finally, the preoperative assessment of esophageal cancer might be strongly impacted by MRI if preclinical data using high power fields are translated into clinical practice, with extremely accurate tumor staging, potentially overtaking endoscopic ultrasonography. MR Esophagography might become a non-invasive, non-irradiating technique of choice following diagnostic esophagogastroduodenoscopy for the assessment of esophageal diseases. 


\section{$\underline{\text { References }}$}

1. Park WG, Shaheen NJ, Cohen J, Pike IM, Adler DG, Inadomi JM, et al. Quality indicators for EGD. Gastrointest Endosc. 2015 Jan;81(1):17-30.

2. ASGE Standards of Practice Committee, Ben-Menachem T, Decker GA, Early DS, Evans J, Fanelli RD, et al. Adverse events of upper GI endoscopy. Gastrointest Endosc. 2012 Oct;76(4):707-18.

3. Schima W. MDCT imaging of the esophagus with sherbet: A step forward. Diagn Interv Imaging. 2016;97(6):591-2.

4. Keulers AR, Hundt W, Mahnken AH. Effect of sherbet timing on distension and evaluation of the esophagus with multidetector-row computed tomography. Diagn Interv Imaging. 2016 Jun;97(6):627-33.

5. Scharitzer M, Pokieser P. What is the role of radiological testing of lower esophageal sphincter function? Ann N Y Acad Sci. 2016 Sep;1380(1):67-77.

6. Hutton B, Salanti G, Caldwell DM, Chaimani A, Schmid CH, Cameron C, et al. The PRISMA extension statement for reporting of systematic reviews incorporating network meta-analyses of health care interventions: checklist and explanations. Ann Intern Med. 2015 Jun 2;162(11):777-84.

7. $\mathrm{Ng} \mathrm{J,} \mathrm{Lee} \mathrm{P.} \mathrm{The} \mathrm{Role} \mathrm{of} \mathrm{Radiotherapy} \mathrm{in} \mathrm{Localized} \mathrm{Esophageal} \mathrm{and} \mathrm{Gastric} \mathrm{Cancer.}$ Hematol Oncol Clin North Am. 2017;31(3):453-68.

8. Yip C, Cook GJR, Landau DB, Davies A, Goh V. Performance of different imaging modalities in assessment of response to neoadjuvant therapy in primary esophageal cancer. Dis Esophagus Off J Int Soc Dis Esophagus. 2016 Mar;29(2):116-30.

9. Schmalfuss IM. Imaging of the hypopharynx and cervical esophagus. Magn Reson Imaging Clin N Am. 2002 Aug;10(3):495-509, vi.

10. Nakashima A, Nakashima K, Seto H, Kakishita M. Normal appearance of the esophagus in sagittal section; measurement of the anteroposterior diameter with ECG gated MR imaging. Radiat Med. 1996 Apr;14(2):77-80.

11. Weijs TJ, Goense L, van Rossum PSN, Meijer GJ, van Lier ALHMW, Wessels FJ, et al. The peri-esophageal connective tissue layers and related compartments: visualization by histology and magnetic resonance imaging. J Anat. 2017;230(2):262-71.

12. Riddell AM, Davies DC, Allum WH, Wotherspoon AC, Richardson C, Brown G. Highresolution MRI in evaluation of the surgical anatomy of the esophagus and posterior mediastinum. AJR Am J Roentgenol. 2007 Jan;188(1):W37-43.

13. Kulinna-Cosentini C, Schima W, Cosentini EP. Dynamic MR imaging of the gastroesophageal junction in healthy volunteers during bolus passage. J Magn Reson Imaging JMRI. 2007 Apr;25(4):749-54. 
14. Manabe T, Kawamitsu H, Higashino T, Lee H, Fujii M, Hoshi H, et al. Esophageal magnetic resonance fluoroscopy: optimization of the sequence. J Comput Assist Tomogr. 2004 Oct;28(5):697-703.

15. Riddell AM, Richardson C, Scurr E, Brown G. The development and optimization of high spatial resolution MRI for imaging the oesophagus using an external surface coil. Br J Radiol. 2006 Nov;79(947):873-9.

16. Ogawa Y, Noda Y, Morio K, Nishioka A, Inomata T, Yoshida S, et al. Ferric ammonium citrate-cellulose paste for opacification of the esophageal lumen on MRI. J Comput Assist Tomogr. 1996 Jun;20(3):455-9.

17. Pavone P, Cardone GP, Cisternino S, Di Girolamo M, Aytan E, Passariello R. Gadopentetate dimeglumine-barium paste for opacification of the esophageal lumen on MR images. AJR Am J Roentgenol. 1992 Oct;159(4):762-4.

18. Kulinna-Cosentini C, Schima W, Lenglinger J, Riegler M, Kölblinger C, Ba-Ssalamah A, et al. Is there a role for dynamic swallowing MRI in the assessment of gastroesophageal reflux disease and oesophageal motility disorders? Eur Radiol. 2012 Feb;22(2):364-70.

19. Kulinna-Cosentini C, Schima W, Ba-Ssalamah A, Cosentini EP. MRI patterns of Nissen fundoplication: normal appearance and mechanisms of failure. Eur Radiol. 2014 Sep;24(9):2137-45.

20. Curcic J, Fox M, Kaufman E, Forras-Kaufman Z, Hebbard GS, Roy S, et al. Gastroesophageal junction: structure and function as assessed by using MR imaging. Radiology. 2010 Oct;257(1):115-24.

21. Roy S, Fox MR, Curcic J, Schwizer W, Pal A. The gastro-esophageal reflux barrier: biophysical analysis on 3D models of anatomy from magnetic resonance imaging. Neurogastroenterol Motil Off J Eur Gastrointest Motil Soc. 2012 Jul;24(7):616-25, e269.

22. Curcic J, Schwizer A, Kaufman E, Forras-Kaufman Z, Banerjee S, Pal A, et al. Effects of baclofen on the functional anatomy of the oesophago-gastric junction and proximal stomach in healthy volunteers and patients with GERD assessed by magnetic resonance imaging and high-resolution manometry: a randomised controlled double-blind study. Aliment Pharmacol Ther. 2014 Nov;40(10):1230-40.

23. Curcic J, Roy S, Schwizer A, Kaufman E, Forras-Kaufman Z, Menne D, et al. Abnormal structure and function of the esophagogastric junction and proximal stomach in gastroesophageal reflux disease. Am J Gastroenterol. 2014 May;109(5):658-67.

24. Faletti R, Gatti M, Di Chio A, Fronda M, Anselmino M, Ferraris F, et al. Concentrated pineapple juice for visualisation of the oesophagus during magnetic resonance angiography before atrial fibrillation radiofrequency catheter ablation. Eur Radiol Exp. 2018 Nov 21;2(1):39.

25. Arnoldner MA, Kristo I, Paireder M, Cosentini EP, Schima W, Weber M, et al. Swallowing MRI-a reliable method for the evaluation of the postoperative gastroesophageal situs after Nissen fundoplication. Eur Radiol. 2018 Nov 12; 
26. Ponds FA, van Raath MI, Mohamed SMM, Smout AJPM, Bredenoord AJ. Diagnostic features of malignancy-associated pseudoachalasia. Aliment Pharmacol Ther. 2017;45(11):1449-58.

27. Panebianco V, Tomei E, Anzidei M, Habib FI, Catalano C, Lisi D, et al. Functional MRI in the evaluation of oesophageal motility: feasibility, MRI patterns of normality, and preliminary experience in subjects with motility disorders. Radiol Med (Torino). 2006 Oct;111(7):881-9.

28. Panebianco V, Habib FI, Tomei E, Paolantonio P, Anzidei M, Laghi A, et al. Initial experience with magnetic resonance fluoroscopy in the evaluation of oesophageal motility disorders. Comparison with manometry and barium fluoroscopy. Eur Radiol. 2006 Sep;16(9):1926-33.

29. Covotta F, Piretta L, Badiali D, Laghi A, Biondi T, Corazziari ES, et al. Functional magnetic resonance in the evaluation of oesophageal motility disorders. Gastroenterol Res Pract. 2011;2011:367639.

30. Miyazaki Y, Nakajima K, Sumikawa M, Yamasaki M, Takahashi T, Miyata H, et al. Magnetic resonance imaging for simultaneous morphological and functional evaluation of esophageal motility disorders. Surg Today. 2014 Apr;44(4):668-76.

31. Nakashima A, Nakashima K, Seto H, Kakishita M, Sakamoto T, Yamada A, et al. Thoracic esophageal carcinoma: evaluation in the sagittal section with magnetic resonance imaging. Abdom Imaging. $1997 \mathrm{Feb}$;22(1):20-3.

32. Wei Y, Wu S, Gao F, Sun T, Zheng D, Ning P, et al. Esophageal carcinoma: Ex vivo evaluation by high-spatial-resolution T2 -mapping MRI compared with histopathological findings at 3.0T. J Magn Reson Imaging JMRI. 2017;45(6):1609-16.

33. Yamada I, Murata Y, Izumi Y, Kawano T, Endo M, Kuroiwa T, et al. Staging of esophageal carcinoma in vitro with 4.7-T MR imaging. Radiology. 1997

Aug;204(2):521-6.

34. Stoker J, van Velthuysen ML, van Overhagen H, van Kempen D, Tilanus HW, Laméris JS. Esophageal carcinoma. Ex vivo endoluminal magnetic resonance imaging. Invest Radiol. 1999 Jan;34(1):58-64.

35. Yamada I, Hikishima K, Miyasaka N, Kawano T, Tokairin Y, Ito E, et al. Esophageal carcinoma: ex vivo evaluation with diffusion-tensor MR imaging and tractography at 7 T. Radiology. 2014 Jul;272(1):164-73.

36. Yamada I, Hikishima K, Miyasaka N, Tokairin Y, Ito E, Kawano T, et al. Esophageal carcinoma: Evaluation with q-space diffusion-weighted MR imaging ex vivo. Magn Reson Med. 2015 Jun;73(6):2262-73.

37. Yamada I, Hikishima K, Miyasaka N, Tokairin Y, Kawano T, Ito E, et al. Diffusiontensor MRI and tractography of the esophageal wall ex vivo. J Magn Reson Imaging JMRI. 2014 Sep;40(3):567-76.

38. Yamada I, Miyasaka N, Hikishima K, Tokairin Y, Kawano T, Ito E, et al. Ultra-highresolution MR imaging of esophageal carcinoma at ultra-high field strength (7.0T) ex 
vivo: correlation with histopathologic findings. Magn Reson Imaging. 2015 May;33(4):413-9.

39. Koyama T, Umeoka S, Saga T, Watanabe G, Tamai K, Kobayashi A, et al. Evaluation of esophageal peristalsis in patients with esophageal tumors: initial experience with cine MR imaging. Magn Reson Med Sci MRMS Off J Jpn Soc Magn Reson Med. 2005;4(3):109-14.

40. Riddell AM, Hillier J, Brown G, King DM, Wotherspoon AC, Thompson JN, et al. Potential of surface-coil MRI for staging of esophageal cancer. AJR Am J Roentgenol. 2006 Nov;187(5):1280-7.

41. Tang Y-L, Zhang X-M, Yang Z-G, Huang Y-C, Chen T-W, Chen Y-L, et al. The Blood Oxygenation T2* Values of Resectable Esophageal Squamous Cell Carcinomas as Measured by 3T Magnetic Resonance Imaging: Association with Tumor Stage. Korean J Radiol. 2017 Aug;18(4):674-81.

42. Wu L, Ou J, Chen T-W, Li R, Zhang X-M, Chen Y-L, et al. Tumour volume of resectable oesophageal squamous cell carcinoma measured with MRI correlates well with T category and lymphatic metastasis. Eur Radiol. 2018 May 14;

43. Giganti F, Salerno A, Ambrosi A, Chiari D, Orsenigo E, Esposito A, et al. Prognostic utility of diffusion-weighted MRI in oesophageal cancer: is apparent diffusion coefficient a potential marker of tumour aggressiveness? Radiol Med (Torino). 2016 Mar;121(3):173-80.

44. Giganti F, Ambrosi A, Esposito A, Del Maschio A, De Cobelli F. Oesophageal cancer staging: a minefield of measurements-author's reply. Br J Radiol. 2017;90(1071):20170054.

45. Liu S, Zhen F, Sun N, Chen J, Cao Y, Zhang S, et al. Apparent diffusion coefficient values detected by diffusion-weighted imaging in the prognosis of patients with locally advanced esophageal squamous cell carcinoma receiving chemoradiation. OncoTargets Ther. 2016;9:5791-6.

46. Giganti F, Ambrosi A, Petrone MC, Canevari C, Chiari D, Salerno A, et al. Prospective comparison of MR with diffusion-weighted imaging, endoscopic ultrasound, MDCT and positron emission tomography-CT in the pre-operative staging of oesophageal cancer: results from a pilot study. Br J Radiol. 2016 Dec;89(1068):20160087.

47. Cardenas ML, Mazur TR, Tsien CI, Green OL. A rapid, computational approach for assessing interfraction esophageal motion for use in stereotactic body radiation therapy planning. Adv Radiat Oncol. 2018 Jun;3(2):209-15.

48. Zhou H-Y, Zhang J-G, Li R, Zhang X-M, Chen T-W, Liu N, et al. Tumour motion of oesophageal squamous cell carcinoma evaluated by cine MRI: associated with tumour location. Clin Radiol. 2018 Jul;73(7):676.e1-676.e7.

49. Heethuis SE, Borggreve AS, Goense L, van Rossum PSN, Mook S, van Hillegersberg R, et al. Quantification of variations in intra-fraction motion of esophageal tumors over the course of neoadjuvant chemoradiotherapy based on cine-MRI. Phys Med Biol. 2018 Jul 19;63(14):145019. 
50. Goense L, Heethuis SE, van Rossum PSN, Voncken FEM, Lagendijk JJW, Lam MGEH, et al. Correlation between functional imaging markers derived from diffusion-weighted MRI and 18F-FDG PET/CT in esophageal cancer. Nucl Med Commun. 2018 Jan;39(1):60-7.

51. De Cobelli F, Giganti F, Orsenigo E, Cellina M, Esposito A, Agostini G, et al. Apparent diffusion coefficient modifications in assessing gastro-oesophageal cancer response to neoadjuvant treatment: comparison with tumour regression grade at histology. Eur Radiol. 2013 Aug;23(8):2165-74.

52. Kwee RM, Dik AK, Sosef MN, Berendsen RCM, Sassen S, Lammering G, et al. Interobserver reproducibility of diffusion-weighted MRI in monitoring tumor response to neoadjuvant therapy in esophageal cancer. PloS One. 2014;9(4):e92211.

53. van Rossum PSN, van Lier ALHMW, van Vulpen M, Reerink O, Lagendijk JJW, Lin $\mathrm{SH}$, et al. Diffusion-weighted magnetic resonance imaging for the prediction of pathologic response to neoadjuvant chemoradiotherapy in esophageal cancer. Radiother Oncol J Eur Soc Ther Radiol Oncol. 2015 May;115(2):163-70.

54. Aoyagi T, Shuto K, Okazumi S, Shimada H, Kazama T, Matsubara H. Apparent diffusion coefficient values measured by diffusion-weighted imaging predict chemoradiotherapeutic effect for advanced esophageal cancer. Dig Surg. $2011 ; 28(4): 252-7$.

55. Imanishi S, Shuto K, Aoyagi T, Kono T, Saito H, Matsubara H. Diffusion-weighted magnetic resonance imaging for predicting and detecting the early response to chemoradiotherapy of advanced esophageal squamous cell carcinoma. Dig Surg. 2013;30(3):240-8.

56. Li Q-W, Qiu B, Wang B, Wang D-L, Yin S-H, Yang H, et al. Prediction of pathologic responders to neoadjuvant chemoradiotherapy by diffusion-weighted magnetic resonance imaging in locally advanced esophageal squamous cell carcinoma: a prospective study. Dis Esophagus Off J Int Soc Dis Esophagus. 2018 Feb 1;31(2).

57. Wang L, Liu L, Han C, Liu S, Tian H, Li Z, et al. The diffusion-weighted magnetic resonance imaging (DWI) predicts the early response of esophageal squamous cell carcinoma to concurrent chemoradiotherapy. Radiother Oncol J Eur Soc Ther Radiol Oncol. 2016;121(2):246-51.

58. Heethuis SE, Goense L, van Rossum PSN, Borggreve AS, Mook S, Voncken FEM, et al. DW-MRI and DCE-MRI are of complementary value in predicting pathologic response to neoadjuvant chemoradiotherapy for esophageal cancer. Acta Oncol Stockh Swed. 2018 Sep;57(9):1201-8.

59. Liu G, Yang Z, Li T, Yang L, Zheng X, Cai L. Optimization of b-values in diffusionweighted imaging for esophageal cancer: Measuring the longitudinal length of gross tumor volume and evaluating chemoradiotherapeutic efficacy. J Cancer Res Ther. 2017;13(5):748-55.

60. Cheng B, Yu J. Predictive value of diffusion-weighted MR imaging in early response to chemoradiotherapy of esophageal cancer: a meta-analysis. Dis Esophagus Off J Int Soc Dis Esophagus. 2018 Jul 16; 
61. Heethuis SE, van Rossum PSN, Lips IM, Goense L, Voncken FE, Reerink O, et al. Dynamic contrast-enhanced MRI for treatment response assessment in patients with oesophageal cancer receiving neoadjuvant chemoradiotherapy. Radiother Oncol J Eur Soc Ther Radiol Oncol. 2016;120(1):128-35.

62. Borggreve AS, Mook S, Verheij M, Mul VEM, Bergman JJ, Bartels-Rutten A, et al. Preoperative image-guided identification of response to neoadjuvant chemoradiotherapy in esophageal cancer (PRIDE): a multicenter observational study. BMC Cancer. 2018 Oct 20;18(1):1006.

63. Zhang J, Hu W, Zang L, Yao Y, Tang Y, Qian Z, et al. Clinical investigation on application of water swallowing to MR esophagography. Eur J Radiol. 2012 Sep;81(9):1980-5.

64. Panebianco V, Francioni F, Anzidei M, Anile M, Rolla M, Passariello R. Magnetic resonance-fluoroscopy as long-term follow-up examination in patients with narrow gastric tube reconstruction after radical esophagectomy. Eur J Cardio-Thorac Surg Off J Eur Assoc Cardio-Thorac Surg. 2006 Oct;30(4):663-8.

65. Erden A, İdılman R, Erden İ, Bektaş M, Özden A. MR angiography of esophageal mural veins in portal hypertension: a correlation with endoscopic grades of esophageal varices. Turk J Gastroenterol Off J Turk Soc Gastroenterol. 2010 Sep;21(3):275-9.

66. Sauter AW, Gatidis S, Schwenzer NF, LA Fougère C, Nikolaou K, Henes JC, et al. Comprehensive metabolic and morphologic disease characterization in systemic sclerosis: initial results using combined positron emission tomography and magnetic resonance imaging. Q J Nucl Med Mol Imaging Off Publ Ital Assoc Nucl Med AIMN Int Assoc Radiopharmacol IAR Sect Soc Of. 2017 03;

Legend to the figures:

Figure 1. Chronologic timeline of major technical developments in MRI of the esophagus.

Figure 2. MRI of the esophagus. Panel A shows a normal esophagus (arrow) on a single shot T2 weighted image, and panel B a widely dilated esophagus (arrow) in a patient with achalasia on steady state weighted image.

Figure 3. Dynamic steady state weighted acquisition in the sagittal oblique plane after administration of water in a 70-year-old patient with a tight fundoplication performed for gastro-esophageal reflux disease 8 years earlier. The fundoplication is responsible for a stenosis of the esophagogastric junction (arrowhead) causing dysphagia and manometric features of achalasia on manometry ("pseudoachalasia"). The oral intake is seen during the swallowing with the dilatation of the oesophagus (arrows) upstream the esophagogastric junction (A-F). The tight fundoplicature (arrows) is well seen on the axial single short T2 weighted images $(\mathrm{G})$. 
Figure 4. 40-year-old woman with dysphagia and regurgitation, and a $5 \mathrm{Kg}$ weight loss. A. Barium esophagogram shows only a mild narrowing of the oeso-gastric junction (arrows) confirmed at endoscopy with no visible endoluminal mass (A). MRI with diffusion weighted sequence in the axial plane $\left(b=1000 \mathrm{~mm}^{2} / \mathrm{s}\right)$ provides additional information regarding presence of liver lesions, which are hemangiomas (arrows) (B). The plane for kinematic acquisition as to be parallel to the esophagogastric junction (arrowheads) (C). T1-weighted image in the coronal plane after intravenous administration of a gadolinium chelate shows a circumferential lesion of 4 centimetres in length in the esophagus wall with mild enhancement (D). Oblique kinematic acquisition with steady state sequence (TruFISP) shows alternatively opening and closing of the esophagus which is narrowed by the circumferential lesion ( $\mathrm{E}$ and F). The circumferential lesion displays a signal typical for leiomyoma.

Figure 5. T3N0 tumor of the esophagogastric junction in 74-year-old man. Circumferential thickening of the esophagogastric junction showing an hypointense signal on T2 weighted images in the coronal plane (A) with marked enhancement of the tissue after administration of gadolinium chelates (B: coronal, D, axial). Restriction of diffusion of the mass on diffusion weighted images in the axial plane $\left(b=1000 \mathrm{~s}^{2} / \mathrm{mm}\right)(C)$. 


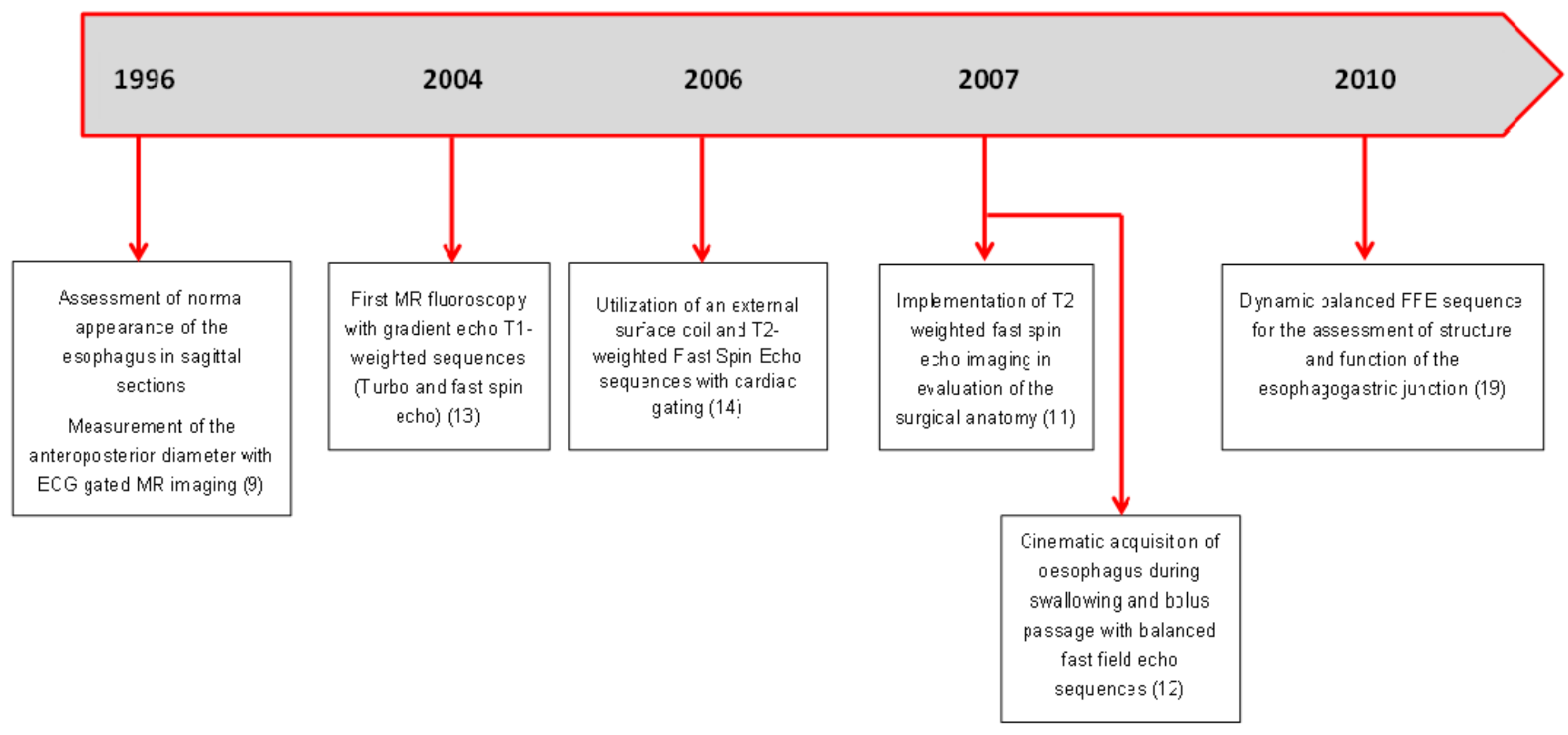




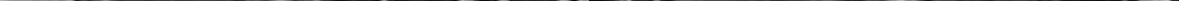




\section{A}

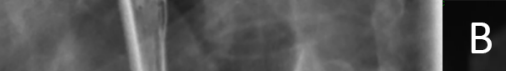

B
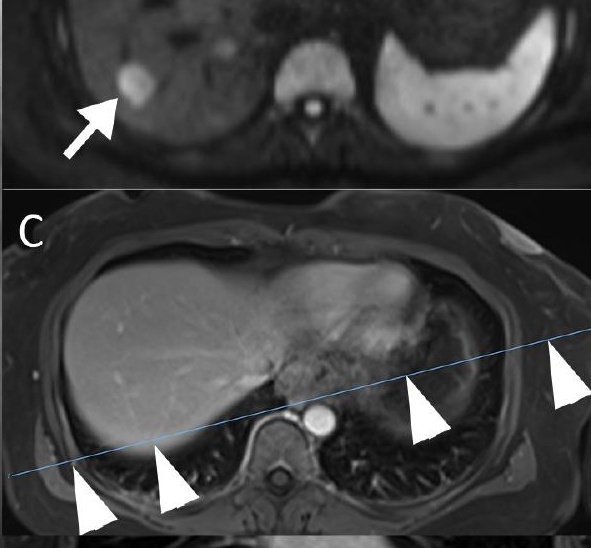

D

E
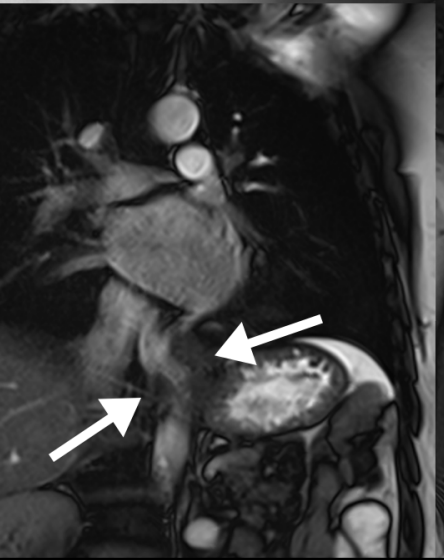

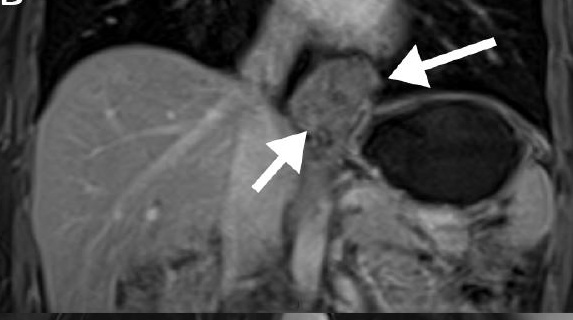

F

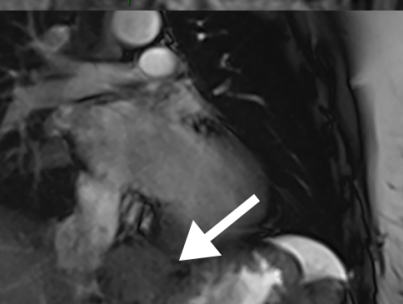

$\lambda\left(\frac{1}{2}\right)$ Fis 


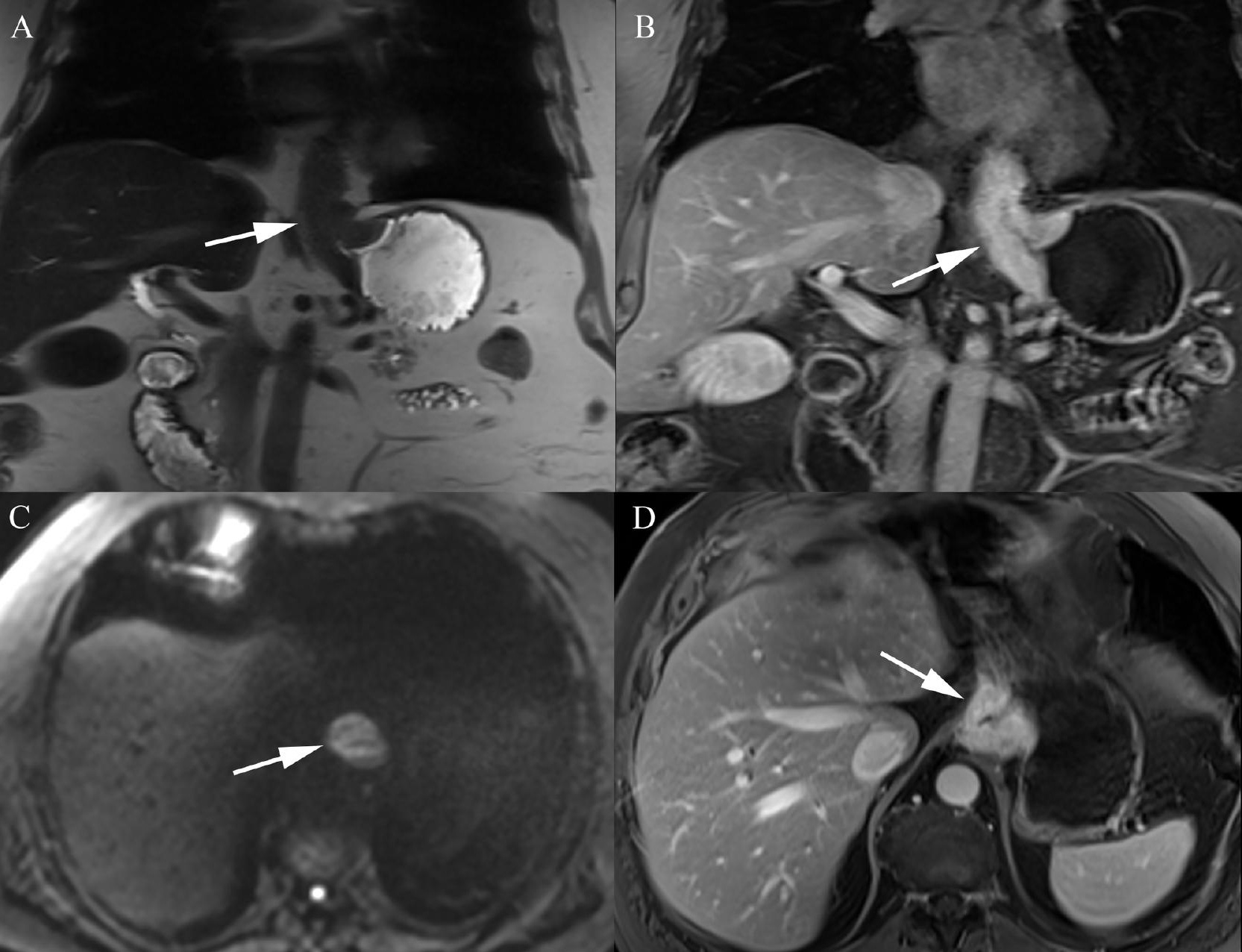


Table 1. MRI sequences parameters at 1.5 Tesla (Siemens Aera, vb20a).

\begin{tabular}{|c|c|c|c|c|}
\hline & $\begin{array}{l}\text { T2-single shot } \\
\text { TSE sequences }\end{array}$ & $\begin{array}{l}\text { Steady states } \\
\text { sequences }\end{array}$ & $\begin{array}{c}\text { Diffusion } \\
\text { sequences } \\
\text { (EPI) }\end{array}$ & $\begin{array}{c}\text { T1-weghted } \\
\text { sequences before } \\
\text { and after IV } \\
\text { administration of } \\
\text { gadolinium- } \\
\text { chelate }\end{array}$ \\
\hline Plane & Axial and coronal & $\begin{array}{l}\text { oblique plane, } \\
\text { parallel to the } \\
\text { eso-gastric } \\
\text { junction }\end{array}$ & Axial & Axial and coronal \\
\hline TE/TR & $93 / 1000 \mathrm{~ms}$ & $1.71 / 433 \mathrm{~ms}$ & $80 / 7900 \mathrm{~ms}$ & $2.19 / 4.85 \mathrm{~ms}$ \\
\hline Flip angle & $150^{\circ}$ & $60^{\circ}$ & $90^{\circ}$ & $10^{\circ}$ \\
\hline FOV & $450 \times 450 \mathrm{~mm}$ & $360 \times 360 \mathrm{~mm}$ & $420 \times 380 \mathrm{~mm}$ & $380 \times 308 \mathrm{~mm}$ \\
\hline $\begin{array}{l}\text { Matrix } \\
\text { size }\end{array}$ & $384 \times 269$ & $256 \times 256$ & $200 \times 200$ & $320 \times 240$ \\
\hline $\begin{array}{l}\text { Slices } \\
\text { thickness }\end{array}$ & $6 \mathrm{~mm}$ & $10 \mathrm{~mm}$ & $7 \mathrm{~mm}$ & $2 \mathrm{~mm}$ \\
\hline Voxel size & $1.2 \times 1.2 \times 6 \mathrm{~mm}^{3}$ & $0.7 \times 0.7 \times 10 \mathrm{~mm}^{3}$ & $1.1 \times 1.1 \times 7 \mathrm{~mm}^{3}$ & $1.2 \times 1.2 \times 2.0 \mathrm{~mm}^{3}$ \\
\hline $\begin{array}{l}\text { Number } \\
\text { of slices }\end{array}$ & 23 & 1 & 40 & 80 \\
\hline $\begin{array}{l}\text { Inter- } \\
\text { slices gap }\end{array}$ & $30 \%$ & NA & $20 \%$ & $20 \%$ \\
\hline
\end{tabular}


Table 2. $\mathrm{b}$ values for Diffusion-weighted imaging (DWI)

b value $\left(\mathrm{s} / \mathrm{mm}^{2}\right)$

De Cobelli et al. Eur Radiol 2013

Imanishi et al. Dig Surg 2013

Kwee et al. PloS One 2014

Yamada et al. Radiology 2014

Van Rossum et al. Oncol 2015

Giganti et al. Br J Radiol 2016

Wang et al. Oncol 2016

Heethuis et al. Radiother Oncom J Eur Soc Ther Radiol Oncol 2016

Liu et al. Oncotarget 2016

Liu et al. J Cancer Res Ther 2017

Wu et al. Eur Radiol 2018

Heethuis et al. Acta Oncol Stockh Swed 2018

Borggreve et al. BMC Cancer 2018
0,600

0,1000

0, 300, 1000

0,1000

$0,200,800$

0,600

0,600

$0,200,800$

0,800

$400,600,800,1000$

$0,500,800$

$0,200,800$

0,800 